pag

Business School

WORKING PAPER SERIES

Working Paper

$2014-251$
L'assemblage dynamique des chaînes logistiques touristiques : vers une réappropriation des activités par les acteurs tunisiens

Frédéric Pellegrin-Romeggio

Dominique Bonet Fernandez

Hatem Trimeche

http://www.ipag.fr/fr/accueil/la-recherche/publications-WP.html

IPAG Business School

184, Boulevard Saint-Germain

75006 Paris

France 


\title{
L'assemblage dynamique des chaînes logistiques touristiques : vers une réappropriation des activités par les acteurs tunisiens
}

Frédéric Pellegrin-Romeggio ${ }^{1}$, Professeur de logistique, Reims Management School, France Dominique Bonet Fernandez ${ }^{2}$, Professeur de supply chain, IPAG Business School, Paris, France

Hatem Trimeche ${ }^{3}$, Maître-assistant en Sciences Economiques, ISG de Sousse, Tunisie.

\section{Résumé}

Le tourisme est souvent considéré comme un facteur essentiel dans la dynamisation d'un territoire tel que la Méditerranée. Bien que la recherche décrive généralement l'industrie touristique sous la forme de différentes chaînes logistiques, il n'existe que peu de travaux sur la problématique de leur assemblage. A travers le concept de l'assemblage dynamique, nous présentons le cas de l'industrie touristique tunisienne, dominée historiquement par des tour operators européens. Nous montrons qu'une réappropriation du secteur par des entreprises tunisiennes est à l'œuvre.

Mots clés : chaîne logistique touristique, assemblage dynamique, Tunisie.

The assembly dynamics of tourism supply chains. Toward a reappropriation of activities by Tunisian actors.

\begin{abstract}
Tourism is often seen as a key factor in boosting a country such as the Mediterranean. Although research generally describes the tourism industry in the form of different supply chains, there is little work on the problem of assembly. Through the concept of dynamic assembly, we present the case of the Tunisian tourism industry, dominated historically by European tour operators. We show that a reappropriation of the sector by Tunisian companies is at work.
\end{abstract}

Keywords: tourism supply chain, dynamic assembly, Tunisia.

Post Print: Maghreb/Machrek, n²16, 2013 /2.

\footnotetext{
${ }^{1}$ CMAC, 59 rue Pierre Taittinger -BP 302, 51061 Reims Cedex. Chercheur affilié au CRET-LOG. frederic.pellegrinromeggio@reims-ms.fr. Tel 0326775561

${ }^{2}$ IPAG LAB, 184, Boulevard St-Germain 75006 Paris, France. dominique.bonet-fernandez@ipag.fr

${ }^{3}$ ISG de Sousse, Rue Abdlaaziz El Behi Bp 763, 4000 Sousse - Tunisie, $\underline{\text { Hatem.trimeche@isgs.rnu.tn }}$
} 


\section{INTRODUCTION}

D’après l'Organisation Mondiale du Tourisme (O.M.T), le tourisme représente près de 10\% du PIB européen et mobilise quelques vingt millions de personnes. Toujours selon l'O.M.T, le bassin méditerranéen reste la première destination touristique mondiale avec $40 \%$ des arrivées de touristes internationaux dans le Monde et $30 \%$ des recettes générées par le Tourisme international. Le Tourisme représente 13\% des exportations des produits méditerranéens et 23\% des activités de services. L’industrie touristique en Méditerranée est donc un enjeu économique primordial (Van der Yeught, 2009 ; Camus et al., 2010).

Depuis une vingtaine d'années les pays MED11 (Algérie, Egypte, Israël, Jordanie, Liban, Libye, Maroc, Autonomie palestinienne, Syrie, Tunisie et Turquie) ont enregistré le plus fort taux de croissance du tourisme mondial entrant. Dans le même temps, le tourisme domestique dans ces pays a été en très forte croissance, malgré les risques de sécurité, les catastrophes naturelles, les hausses des prix du pétrole et les incertitudes économiques de cette zone.

En 2010, l'industrie du tourisme des 11 pays MED, représentait directement et indirectement $10,9 \%$ du PIB en moyenne, confirmant son importance économique majeure pour ces pays. Les parts des pays variaient d'un maximum de $20,4 \%$ en Jordanie à un minimum de $6,3 \%$ en Israël. L'emploi touristique direct et indirect contribue à l'emploi total dans les pays MED 11 (13.58\% du total en 2010). Toutefois, la croissance de l'emploi touristique est plus lente que l'augmentation du nombre d'arrivées de touristes internationaux.

La dernière crise financière a eu des conséquences graves sur ce développement, qui a confirmé la résilience du tourisme et de l'énorme potentiel des 11 pays MED dans ce secteur. Cette tendance a été brusquement interrompue au début de 2011 (Cf tableau 1) au cours du printemps arabe, mais pourrait reprendre lorsque la situation se stabilisera.

Tableau 1 : La récession du secteur touristique post révolution

\begin{tabular}{|c|c|c|c|c|c|}
\hline Paramètres & 2009 & 2010 & 2011 & $\begin{array}{r}\text { Ecart en \% } \\
\text { 2011/2010 }\end{array}$ & $\begin{array}{l}\text { Ecart en \% } \\
2011 / 2009\end{array}$ \\
\hline $\begin{array}{l}\text { Recettes touristiques } \\
\text { en MDT }\end{array}$ & $3,471.9$ & 3.522 .5 & 2.364 .5 & $-32,9$ & $-31,9$ \\
\hline Nuitées globales & $34.623,504$ & 35.565 .104 & 21.236 .067 & $-40,3$ & $-38,7$ \\
\hline Arrivées aux frontières & 6.901 .406 & 6.902 .749 & 4.781 .896 & $-30,7$ & $-30,7$ \\
\hline
\end{tabular}

Source : Direction des études du Ministère du tourisme tunisien (http://www.tourisme.gov.tn)

Cette croissance MED tourisme 11 contraste avec le faible développement du tourisme dans les pays membres de l'UE de la Méditerranée nord au cours de la même période. Les 11 pays MED ont lancé des programmes de développement ambitieux après 1995, et les dépenses publiques en matière de tourisme ont quadruplé entre 1990 et 2010. Néanmoins, le niveau d'investissement en capital peut-être augmenté pour assurer le développement compétitif et durable de ce secteur. Les indicateurs montrent que les pays MED 11 se retrouvent en bas du 
classement au niveau international dans tous les sous-indices concernant le tourisme, à savoir le cadre réglementaire, l'environnement des affaires et de l'infrastructure et des ressources humaines, culturelles et naturelles dans le tourisme.

Nous utilisons ici la définition du tourisme utilisée par l'Organisation mondiale du tourisme (OMT) comme la somme des secteurs comme la construction et les infrastructures, le transport, l'hébergement, la nourriture et les services de restauration, de loisirs et de divertissement, les agences de voyages ou les tour-opérateurs et une part importante des activités de l'artisanat, de la culture et du patrimoine.

Au cours des 20 dernières années, le tourisme méditerranéen a été dominé par l'évolution de la rive nord de la Méditerranée. Cet article s'interroge sur cette tendance et sur le rôle des acteurs intégrateurs des services touristiques que sont les tour operators, en Tunisie.

L'article procède en quatre étapes. Dans une première étape, le cadre théorique est élaboré en référence au concept d'assemblage dynamique des services touristiques. Dans une seconde section, nous présentons la méthodologie. La troisième partie est consacrée à l'étude de cas et la quatrième partie à la discussion du rôle des TO en Tunisie. Les résultats obtenus nous permettent de souligner l'émergence d’une réappropriation des activités intégratrices du tourisme par les acteurs tunisiens.

\section{Les concepts de Tourism Supply Chain Management et d'assemblage dynamique : vers un management innovant des réseaux touristiques}

En réponse à l'accroissement des besoins en réactivité et en flexibilité des différents acteurs, la dimension dynamique dans le management des réseaux et des chaînes logistiques touristiques devrait être davantage prise en compte. Le concept innovant d'assemblage dynamique, relié à celui du Tourism Supply Chain Managment (TSCM) va dans ce sens.

Les premières études sur les chaînes logistiques dans le tourisme (TSC) apparaissent au début des années 1990 (UNWTO ${ }^{4}, 1994$; Sinclair et Stabler 1997 ; Buhalis et Law, 2001 ; Page, 2003), mais demeurent essentiellement des études descriptives. Plus tard, Tapper et Font (2004) définissent la supply chain touristique (TSC) et la distinguent des supply chains des autres secteurs. Ils soulignent que la principale différence entre la supply chain touristique et celles des autres secteurs est que les touristes voyagent dans le produit et que la plupart des composants du produit sont des services, et aussi que la qualité des vacances pour le consommateur dépend de la performance de tous les maillons de la supply chain touristique.

\footnotetext{
${ }^{4}$ UNWTO : United Nations World Tourism Organization
} 
Figure 1- Les acteurs de la supply chain touristique

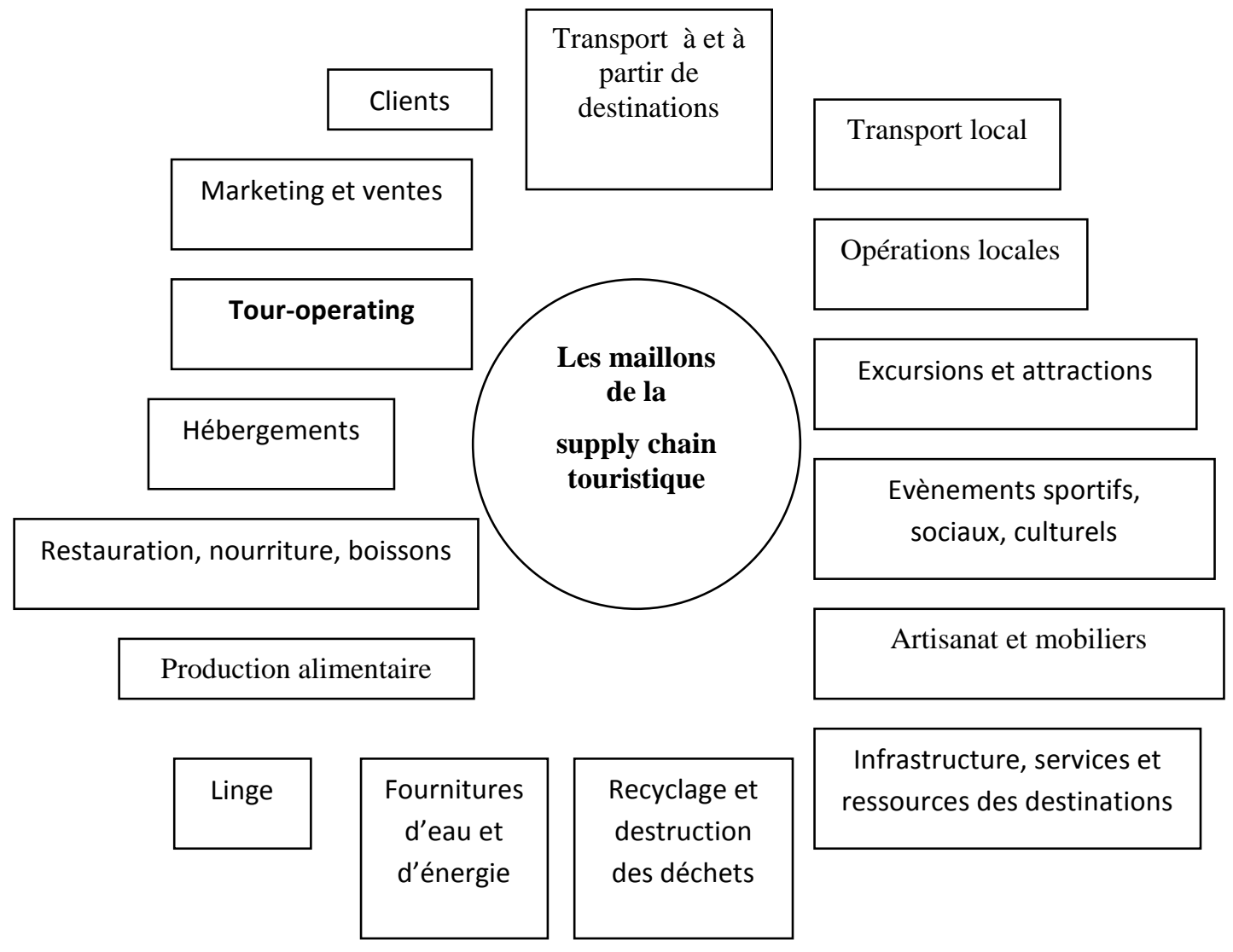

Source : schéma inspiré de Tapper et Font (2004), p.4

Ce schéma, inspiré de Tapper et Font (2004), montre la configuration réticulaire et multiacteurs de la chaîne touristique, et par analogie, de celle du voyage. Mais au-delà de l'approche uniquement descriptive des supply chains touristiques, il convient d'analyser également le management spécifique de ce type de chaînes logistiques, avec le concept de Tourism Supply Chain Management (TSCM). Zhang et al. (2009, p.3) sont probablement les premiers à proposer une définition précise et explicite du concept de tourism supply chain management (TSCM). Ces auteurs présentent le TSCM comme une véritable philosophie de management, visant à coordonner une chaîne logistique multi-acteurs vers des objectifs partagés et une collaboration renforcée. D’une manière générale, Zhang et al. (2009) distinguent le secteur du tourisme des autres secteurs économiques par six caractéristiques :

- Le tourisme est une industrie avec une coordination intensive dans laquelle les différents produits/services sont regroupés dans des packages (bundled together).

- $\quad$ Les produits touristiques ne peuvent être stockés, ils sont périssables.

- Puisque les produits touristiques ne peuvent être testés avant leur consommation, ils dépendent fortement de leur présentation et de l'interprétation des clients et des vendeurs, donc l'industrie touristique est «informationnellement dépendante » et liée à une information intensive.

- Les produits touristiques sont complexes par nature, ils sont hétérogènes et composés à partir de nombreux composants de services différents.

- L’industrie du tourisme doit faire face à une forte demande, mais incertaine, 
- L'industrie du tourisme s'inscrit dans une structure organisationnelle extrêmement dynamique, avec une forte concurrence parmi les prestataires de services. Cette structure dynamique permet aux acteurs de changer de partenaires commerciaux en fonction des besoins (capacités de substitution).

D’après Zhang et al. (2009) ces caractéristiques ne sont pas suffisamment prises en compte dans la littérature scientifique pour le management "dynamique » des chaînes logistiques touristiques. Sur la base, notamment, de ces constatations, nous proposons donc un nouveau cadre conceptuel pour le management des chaînes logistiques multiservices, permettant d’intégrer à la fois la notion d'assemblage ainsi que sa dimension dynamique.

\section{Méthodologie de recherche}

La nature de l'objet de notre recherche nous a conduit à privilégier une approche qualitative basée sur une étude de cas unique. Yin (2003) distingue l'étude de cas unique et l'étude de cas multiples. Afin d'accroître la validité interne et externe de l'étude de cas, Yin (2003) suggère concernant le cas unique, de recourir davantage à la théorie, comme par exemple la conceptualisation, et d'utiliser la réplication pour les cas multiples. L'apparition récente du phénomène de l'assemblage dynamique nous conduit à analyser avant tout la pratique des acteurs dans un contexte précis. Nous avons donc privilégié une étude de cas unique approfondie plus en rapport avec la dimension " exploratoire » de notre recherche. Notre travail repose sur une recherche empirique menée en 2012 en Tunisie, qui s’appuie sur l'analyse de documents professionnels et académiques, complétée par une série d'entretiens et d'observations chez une vingtaine d'acteurs de l'industrie du voyage. Pour le recueil et le traitement des données primaires et secondaires, nous avons procédé à une analyse de contenu thématique. Nous avons composé quatre dimensions du concept d'assemblage dynamique : conception, combinaison, coordination, contrôle.

Figure 2- La chaîne logistique du voyage

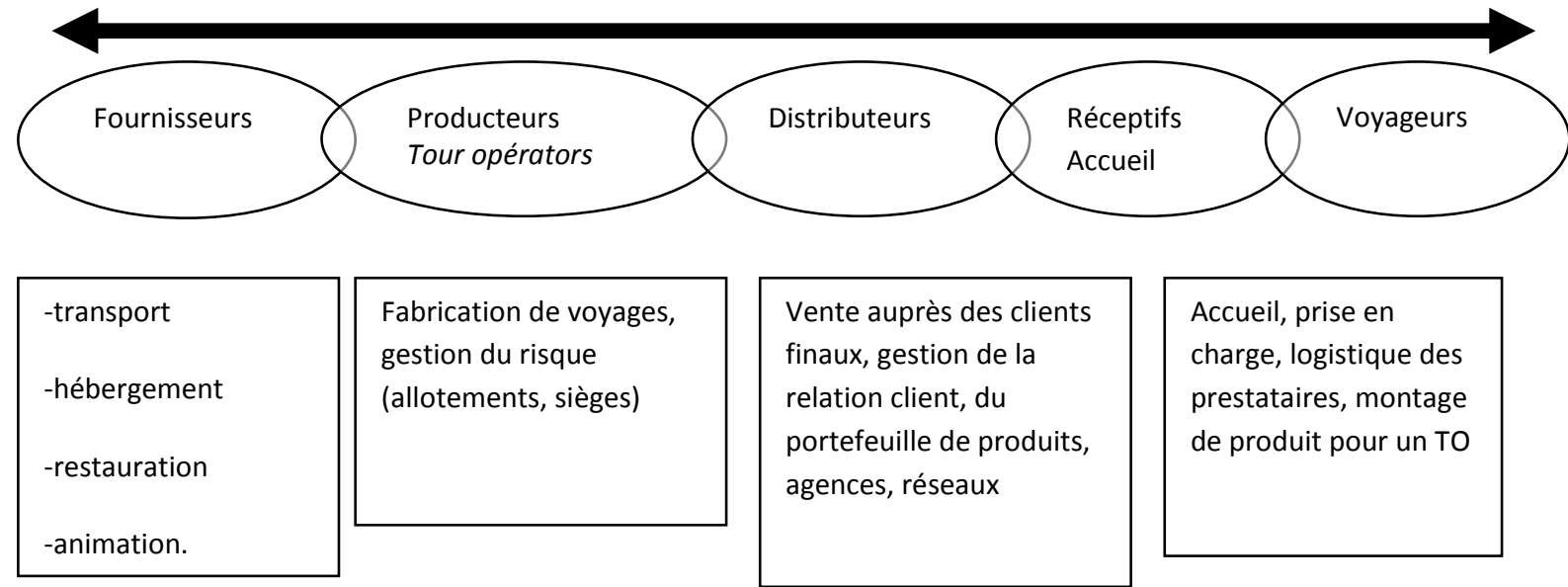

\section{Modèle d'assemblage des services touristiques en Tunisie, le rôle du TO}

La politique touristique de la Tunisie a historiquement était tournée vers la standardisation des services. Il s'agit de séjours à bas prix sous forme de pack voyage. Les pouvoirs publics ont 
adopté une politique de pénétration avec une vente massive de produits homogènes à bas prix. Avec 850 unités hôtelières et 241.000 lits, la Tunisie présente une capacité d'accueil très importante avec une évolution exponentielle durant ses vingt dernières années. La Tunisie dispose d'une compétitivité hors coût par la structure de l'offre (proximité géographique de l'Europe, climat doux, pays francophone, et offre très importante). Les produits touristiques, les séjours nécessitent une organisation commerciale et marketing. A ce niveau interviennent les Tours Operators (TO) pour organiser l'offre de services touristiques entre les clients (non résidents) et les prestataires de services locaux.

La commercialisation des services touristiques se fait comme suit. Le TO s'adresse au Contact Manager de l'hôtel afin d'établir une convention, qui consiste à établir un accord dont les services fournis et le prix représentent les principaux éléments de l'accord. Le TO recrute un représentant local appelé « agence réceptive », il s'agit souvent d'une agence locale de voyage qui met à la disposition du TO des bureaux pour exercer ses activités.

Le TO met à la disposition du client un service composé de cinq étapes :

1. Le transport aérien

2. Le transfert aéroport / hôtel

3. Séjour selon la formule choisie comme indiquée dans le voucher

4. Une formule d'excursion (Sahara, découverte, Tabarka), il s'agit d'une formule optionnelle choisie au moment de l'achat du pack ou au cours du séjour en s'adressant à l'agence réceptive.

5. Le transfert hôtel / aéroport

Le tourisme tunisien s'inscrit ainsi dans le modèle de fonctionnement classique des TO basé sur l'assemblage dynamique des services. Ce modèle est probablement en phase de déclin dans certains autres pays, au profit du self-packaging sur des sites on line. $\mathrm{Vu}$ la structure et les spécificités actuelles du secteur en Tunisie, le self-packaging ne peut pas se développer pour deux raisons :

1. Le pouvoir de négociation des TO reste le moyen exemplaire pour obtenir des séjours à prix attractifs. Les TO sont les seuls à fournir des séjours quasiment au simple prix du vol.

2. Il n’existe pas réellement d'autre alternatives au tourisme balnéaire proposé par les TO (95\% des lits), les tourismes culturel, saharien, rural, écologique, de croisière restent relativement peu développés. Par contre le tourisme médical se développe d'une manière prometteuse avec $150.000^{5}$ patients étrangers ayant séjourné dans les cliniques tunisiennes ${ }^{6}$ en 2007. Le client est donc face à un marché de concurrence monopolistique avec des produits homogènes. Le client a intérêt à choisir ce système car c'est le seul qui lui offre des prix intéressants.

Le TO, "pivot » est donc la pièce maîtresse dans l'assemblage des prestations fournies par le secteur touristique tunisien. La combinaison, est une contrainte dans la mesure où la diversité de l'offre et des modalités des services imposent l'élaboration d'une combinaison optimale à partir des ressources territoriales pour former un panier de services capable de toucher des

\footnotetext{
${ }^{5}$ Les Chiffres de l’Union Tunisienne de l'Industrie du commerce et de l'Artisanat (UTICA)

${ }^{6}$ Nous distinguons deux types de services :

- $\quad$ Chirurgie esthétique, destinée principalement aux patients européens.

- Les soins classiques destinés aux patients maghrébins fortunés, l'infrastructure médicale dans leur pays reste relativement modeste.
} 
catégories socioprofessionnelles diversifiées. Ainsi le rôle du pivot est de présenter d'une manière optimale un potentiel de ressources spécifiques construit localement. Les ressources territoriales sont amplifiées et combinées par les TO pour être présentées sous la forme actuelle des services touristiques. La chaîne logistique du voyage mentionnée dans le tableau 2, représente le modèle d'assemblage dynamique des services touristiques en Tunisie.

Tableau 2 : La chaîne logistique des services touristiques en Tunisie

\begin{tabular}{|c|c|c|c|c|c|c|c|}
\hline \multicolumn{8}{|c|}{$\begin{array}{l}10 \text { tours opérateurs animent le tourisme tunisien (absorbant les 2/3 de l'offre) } \\
\qquad \underline{\text { 10 Pivots }}\end{array}$} \\
\hline Etape 1 & \multicolumn{7}{|c|}{ Accord sur les services d'une manière individuelle } \\
\hline & \multicolumn{2}{|l|}{ Vol } & Transfert & Hébergement & \multicolumn{2}{|r|}{ Animation } & Excursion \\
\hline & \multicolumn{2}{|c|}{$\begin{array}{l}\text { Etablir des conventions de } \\
\text { remplissage des appareils } \\
\text { avec les compagnies } \\
\text { locales : Tunisair, } \\
\text { Nouvelair ou avec des } \\
\text { compagnies étrangères } \\
\text { Exemple : Nouvelair est le } \\
\text { transporteur de Marmara }\end{array}$} & $\begin{array}{l}\text { L'agence } \\
\text { réceptive } \\
\text { fournit } \\
\text { l'assistance au } \\
\text { client à } \\
\text { l'aéroport ainsi } \\
\text { que le transfert } \\
\text { à l'hôtel }\end{array}$ & $\begin{array}{l}\text { Convention avec les } \\
\text { hôteliers sur les } \\
\text { modalités } \\
\text { d’hébergement } \\
\text { (LPD/DP/PC ou All } \\
\text { inclusive) ainsi que } \\
\text { sur les tarifs. }\end{array}$ & \multicolumn{2}{|c|}{$\begin{array}{l}\text { Activités sportives (ski } \\
\text { nautique, parachute, golf) } \\
\text { ou divertissement de } \\
\text { jeunesse (discothèques ou } \\
\text { concerts) }\end{array}$} & $\begin{array}{l}\text { Accords avec les agences } \\
\text { locales pour fournir à la } \\
\text { demande des excursions } \\
\text { sahariennes, culturelles } \\
\text { (mausolées, } \\
\text { amphithéâtres romains) } \\
\text { ou maritimes (sorties en } \\
\text { mer sur des bateaux } \\
\text { pirate). }\end{array}$ \\
\hline & \multicolumn{2}{|c|}{$\begin{array}{l}\text { Service fondamental pour } \\
\text { le montage du produit }\end{array}$} & $\begin{array}{l}\text { Service } \\
\text { fondamental } \\
\text { pour le montage } \\
\text { du produit }\end{array}$ & $\begin{array}{l}\text { Service fondamental } \\
\text { pour le montage du } \\
\text { produit }\end{array}$ & \multicolumn{2}{|c|}{$\begin{array}{l}\text { Service optionnel mais } \\
\text { pouvant être un outil } \\
\text { marketing surtout s'il est } \\
\text { offert par le TO }\end{array}$} & $\begin{array}{l}\text { Service optionnel mais } \\
\text { pouvant être un outil } \\
\text { marketing surtout s'il est } \\
\text { offert par le TO }\end{array}$ \\
\hline \multirow[t]{2}{*}{ Etape 2} & \multicolumn{7}{|c|}{ Assemblage et combinaison des services } \\
\hline & Produit 1 & \multicolumn{2}{|c|}{ Produit 2} & \multicolumn{2}{|l|}{ Produit 3} & Produit 4 & Produit 5 \\
\hline \multirow[t]{2}{*}{ Critères } & $\begin{array}{l}\text { Région, période, } \\
\text { budget, âge, services } \\
\text { fournis etc }\end{array}$ & \multicolumn{2}{|c|}{$\begin{array}{l}\text { Région, période, budget, } \\
\text { âge, services fournis etc }\end{array}$} & \multicolumn{2}{|c|}{$\begin{array}{l}\text { Région, période, budget, } \\
\text { âge, services fournis etc }\end{array}$} & $\begin{array}{l}\text { Région, période, } \\
\text { budget, âge, services } \\
\text { fournis etc }\end{array}$ & $\begin{array}{l}\text { Région, période, } \\
\text { budget, âge, services } \\
\text { fournis etc }\end{array}$ \\
\hline & $\begin{array}{l}\text { Catégorie } \\
\text { socioprofessionnelle } \\
1\end{array}$ & \multicolumn{2}{|c|}{$\begin{array}{l}\text { Catégorie } \\
\text { socioprofessionnelle } 2\end{array}$} & \multicolumn{2}{|c|}{$\begin{array}{l}\text { Catégorie } \\
\text { socioprofessionnelle } 3\end{array}$} & $\begin{array}{l}\text { Catégorie } \\
\text { socioprofessionnelle } \\
4\end{array}$ & $\begin{array}{l}\text { Catégorie } \\
\text { socioprofessionnelle } \\
5\end{array}$ \\
\hline \multirow[t]{2}{*}{ Etape 3} & \multicolumn{7}{|c|}{ Commercialisation et distribution des produits } \\
\hline & \multicolumn{3}{|l|}{ Moyens propres des TO } & hysiques & Le $n$ & : Comparateur des séj & ours et voyagistes virtuels \\
\hline Etape 4 & \multicolumn{7}{|c|}{ Vente du produit et encaissement } \\
\hline Etape 5 & \multicolumn{7}{|c|}{ Consommation du produit } \\
\hline Etape 6 & \multicolumn{7}{|c|}{ Solde des comptes avec les différents fournisseurs de services } \\
\hline
\end{tabular}

Le rôle des TO dans la relance économique ne fait aucun doute. Avec plus de 7\% du PIB et plus de 400.000 emplois, le secteur touristique, principalement animé par les TO, est un pilier de l'économie tunisienne. Néanmoins, l'intervention massive des TO dans le secteur touristique tunisien lui confère une position de force dans les processus de négociation et d'organisation des services. Cependant, cette success story a soulevé un certain nombre de critiques, principalement d'ordre déontologique et moral.

Les TO opérateurs imposent leurs conditions aux hôteliers, qui, pour survivre dans un milieu très concurrentiel, n'ont que le choix de coopérer. Outre les prix écrasés (atteignant 7 euros la nuit en basse saison), les TO exigent la gratuité de certains services et prestations utilisés ultérieurement comme des atouts de marketing sur leurs brochures et prospectus. Le paiement des services par les TO s'effectue à terme. En effet le client paye son séjour quelques mois ou quelques semaines avant l'échéance, par contre l'hôtelier ne sera payé que quelques mois après l'échéance. Ce modèle de 
fonctionnement est défavorable au secteur hôtelier tunisien, fragilisé par un endettement important et des délais de paiement trop longs de la part des TO.

La domination des hôteliers par les TO a créé trois scénarii.

- Une nouvelle expérience en matière de concentration des services sous une seule enseigne: Nouvelair (transport aérien), Tunisian Travel Service (transport terrestre) et quatre unités hôtelières appartenant au groupe (Phénicia Hotel à Hammamet, Sahara Beach à Monastir, Sahara Douz Hotel à Douz et Djerba Beach Hotel à Djerba) font partie de la même holding. La concentration des services sous une seule enseigne ne résout pas le problème de la dépendance vis-à-vis d'un TO qui est le seul capable de vendre les prestations, même fournies par la holding.

- En 2012, El Mouradi, une grande chaîne d'hôtel en Tunisie réunissant 17 hôtels et 16000 lits, a décidé de créer son propre TO. "Sunshine Vacances », premier TO tunisien, opérant principalement sur le marché français.

- La contre-attaque des TO à partir de 2009, a consisté à limiter la dépendance des TO vis-à-vis des hôteliers locaux. Désormais, les TO louent et gèrent des hôtels afin d'y faire séjourner leurs clients. Cette politique s'avère efficace, elle a été principalement adoptée par Thomas Cook et Marmara.

Le quasi-monopole ${ }^{7}$ des TO et leur position de force les rend plus exigeants sur la qualité de service. Le pivot modernise indirectement le secteur en exigeants climatisation, piscines couvertes, chaines satellitaires, terrains de tennis, salons de coiffure, centres de fitness et même des efforts écologiques. Certes, c'est à l'avantage du TO, mais cela améliore considérablement la compétitivité du tourisme tunisien dans le bassin méditerranéen. Cette attitude coïncide avec le programme de mise à niveau des hôtels qui a commencé en 2005. A ce jour 108 hôtels ont pu en bénéficier.

Dans ces conditions, les orientations futures de la politique touristique tunisienne demeurent.

- Une reconnaissance vis-à-vis du soutien des TO français qui restent les plus actifs après la révolution tunisienne, au moment où les autres opérateurs étaient réticents et septiques sur la capacité de la Tunisie à surmonter les problèmes sécuritaires post révolution : 1,4 million de français ont visité la Tunisie en 2011. On assiste d'ailleurs à l'implantation du groupe Accor dans le pays avec deux unités déjà inaugurées (Ibis Tunis et Novotel Tunis), et l'ouverture d'une vingtaine d'autres hôtels (entre 20 et 25 selon la demande) d'ici quelques années.

- $\quad$ L’idée dominante était de profiter du soutien des TO français pour restructurer le secteur, la fédération nationale de l'aviation marchande (FNAM), le Syndicat national des agences de voyages (SNAV), l'association des tours opérateurs français (CETO).

- Le soutien des TO se caractérise par l'engagement de ne pas brader les prix sur la Tunisie alors que les TO étaient en position de force pour le faire, dans un contexte de post révolution caractérisé par le ralentissement des activités économiques et le détournement des séjours au profit du Maroc et de la Turquie.

- La mission des «pivots » est une véritable prise de participation dans la relance d’un secteur en crise dans un pays en difficulté.

\footnotetext{
${ }^{7}$ 70\% des touristes allemands ont séjourné en Tunisie grâce aux services de trois TO allemand. Les 2/3 des clients français sont envoyés par cinq TO. Egalement les 2/3 des clients britanniques sont envoyés par deux TO.
} 
- Néanmoins, les TO deviennent de plus en plus exigeants sur les conditions des séjours (enfant - de 6 ans gratuit, plus de propreté, supplément single gratuit, meilleure restauration...)

- La future stratégie des politiques est d'occuper les places médiatiques françaises via des spots publicitaires. Cette stratégie est appliquée depuis des années par la Turquie et le Maroc. Elle s'est montrée d'une grande efficacité.

- Les côtes tunisiennes considérées comme ressources spécifiques territoriales devraient être exploitées d'une manière optimale avec la création en 2015 de quatre nouvelles stations balnéaires.

\section{Discussion : une confirmation empirique de l'assemblage dynamique dans l'industrie du voyage en Tunisie}

Les résultats de notre recherche confirment la réalité de l'assemblage dynamique dans l'industrie du voyage en Tunisie à travers la mobilisation des quatre dimensions (conception, combinaison, coordination, contrôle) avec toutefois certaines nuances qu'il convient de relever.

Au niveau de la dimension conception, le pivot assembleur a démontré sa capacité à repérer, à sélectionner et à positionner des acteurs potentiellement activables, même si la chaîne du voyage apparaît davantage comme une chaîne de compétences clés que comme une simple chaîne d'acteurs figés aux rôles définitifs (fournisseurs, producteurs, distributeurs, etc.). Egalement, le pivot assembleur a la compétence pour construire la structure organisationnelle au sein de laquelle il activera les chaînes logistiques multiservices. En effet, le pivot assembleur, historiquement le tour operator, mais de plus en plus un quelconque acteur " désintermédié » et compétent a en charge la mobilisation de prestataires potentiels au sein d'un réseau essentiellement préconçu. Le pivot assembleur joue un rôle de facilitateur relationnel, au sein d'un réseau relativement stable et pérenne. Enfin, l'impact de la technologie et d'Internet est capital sur les modalités de construction de la supply chain du voyage, en influençant notamment les politiques de commercialisation multicanal. Par conséquent, la dimension conception est réellement mobilisé par le pivot assembleur, dans la mesure où elle vise à analyser sa capacité à établir les fondements structurels, le design général de la structure organisationnelle ainsi qu’à définir le niveau d’intégration souhaité.

Au niveau de la dimension combinaison, la capacité du pivot assembleur à combiner au gré des besoins en différentes prestations se manifeste clairement à travers des éléments visibles. Le premier d'entre eux correspond sans doute à l'existence de normes ou de standards internationaux que l'on retrouve à la fois dans la sphère technologique et dans celle de la qualité (ex : ISO, HACCP, JAR, IOSA). L'existence de standards permet aux uns et aux autres de se repérer, de se positionner par rapport aux niveaux de prestations recherchées, de se sécuriser, et de rester cohérent. La mise en œuvre de ces standards ou normes se concrétise principalement par la maîtrise d'interfaces technologiques, mais également par la gestion d'interfaces relationnelles. Par ailleurs, la volonté à donner toujours plus de choix aux consommateurs est récurrente chez la majorité des acteurs observés. Cela soulève inévitablement la problématique de la gestion des stocks à travers des enjeux évidents de disponibilité et de flexibilité de l'assemblage. Mais à défaut de proposer systématiquement des produits à la carte ou complètement personnalisés et flexibles, tous s'accordent à reconnaître qu'on peut moduler plus ou moins les prestations à partir de composants de base qu’on agence au gré des besoins et des possibilités d'assemblage. 
La dernière dimension du concept d'assemblage dynamique se manifeste en premier lieu avec un contrôle ex ante relatif aux capacités et ressources à mettre en œuvre, suivi d'un contrôle ex post visant à évaluer les différentes prestations réalisées. Concernant le contrôle ex ante, nos résultats montrent l'importance de l'anticipation des besoins à travers une évaluation des capacités ou des ressources nécessaires à la réalisation de la prestation. Dans le cas des assembleurs qui ont fait le choix du volume et de la domination par les coûts et donc les prix, la réservation des capacités par avance est une contrainte réelle, car il s'agit là d'une problématique classique de gestion des stocks. Dans le cas des assembleurs qui se différencient par la spécialisation et le sur mesure, il s'agit davantage d'évaluer par avance des capacités potentielles, activables, avec le risque majeur d'une absence de disponibilité au moment de la demande. La maîtrise de l'information en temps réel est ici indispensable car elle permet cette vision globale et instantanée.

En dehors et en complément du cas de l'industrie du voyage, notre recherche pourrait être menée au niveau des territoires et donc des destinations touristiques. Par ailleurs, au-delà de la sphère touristique ce travail laisse entrevoir de nombreuses perspectives, notamment pour les supply chains temporaires et certaines organisations type projet. Notre travail pourrait être étendu à l'ensemble des supply chains temporaires (De Waard et Kramer, 2008) et à certaines organisations configurées en mode projet comme par exemple les chaînes saisonnières ou la logistique événementielle (ex : spectacles, manifestations, etc.). Notre travail pourrait se prêter également à la réplication à travers des études de cas multiples (Pays MED 11), (Yin, 2003), ce qui renforcerait la validité interne et externe de notre recherche et donc sa généralisation. Cette réplication de nos travaux pourrait être conduite dans d'autres secteurs des services comme par exemple la logistique humanitaire (Larsson et Vega Bernal, 2010), les prestataires de services logistiques (PSL) (Kacioui-Maurin, 2010) mais également dans l'industrie avec des entreprises qui travaillent en mode projet (ex : dans la confection, Dari, 2010).

\section{Conclusion}

Les résultats de notre recherche nous conduisent aux conclusions suivantes. L’industrie du voyage en Tunisie mobilise différentes chaînes logistiques touristiques dans lesquelles la problématique de l'assemblage des ressources est essentielle. Ces assemblages requièrent des dispositifs plus ou moins souples, modulaires et dynamiques, ainsi qu'une compétence spécifique d'assembleur pour les pivots des différents réseaux centrés d'acteurs. Une telle compétence ne peut être mobilisée efficacement pour l'assemblage dynamique des chaînes logistiques qu'à travers la maîtrise de quatre dimensions : la conception, la combinaison, la coordination, et le contrôle des structures organisationnelles mises en place. D'un point de vue managérial, notre recherche démontre que les acteurs locaux mettent progressivement en œuvre leurs propres solutions. Cette compétence particulière constitue ainsi une nouvelle forme d'innovation managériale qui pourrait être appliquée notamment pour la gouvernance des territoires.

La mission des "pivots » revêt une véritable participation dans la relance d'un secteur en crise dans un pays en difficulté. Il est important de noter qu'à partir des années 2000, et avec l'amélioration notable du niveau de vie moyen des tunisiens, les hôteliers se sont tournés vers le marché local ce qui réduit partiellement la dépendance de ces derniers envers les TO. Ainsi les produits se vendent via les agences physiques et les sites online (traveltodo.com, Elmouradi.com). Une tendance vers la réappropriation de l'industrie touristique par les acteurs locaux est à l'œuvre. Dans un contexte institutionnel tunisien favorable au développement touristique, la Tunisie pourrait montrer la voie aux autres pays de la Méditerranée. 


\section{Bibliographie}

Buhalis, D., Law, E. (2001). Tourism distribution channels: Practices, issues and transformation. London: Continuum International Publishing Group.

Camus, S., Hikkerova, L., Sahut, J.M. (2010), "Tourisme durable : une approche systémique ", Cahier : Vers un Tourisme Durable ou un écotourisme, No 34, Management \& Avenir, avril.

Dari, L. (2010), Le rôle des acteurs tiers dans les relations inter organisationnelles : le cas du secteur du prêt-à-porter, Thèse de Doctorat en Sciences de Gestion, Université de la Méditerranée, Aix-Marseille II, soutenue le 20 septembre.

De Waard, E.J., Kramer, E.H., (2008), “Tailored task forces: Temporary organizations and modularity”, International Journal of Project Management, Vol. 26, n5, pp.537-546.

Kacioui-Maurin, E. (2010), " L'innovation chez les PSL, évolutions, enjeux et perspectives », 8th International Meeting for Research in Logistics (RIRL), Colloque organisé par BEM Bordeaux Management et RIRL, Bordeaux, octobre.

Larsson, F.E., Vega Bernal, D. (2010), "Green logistics in temporary organizations: A paradox? Learnings from the humanitarian context”, The 8th International Conference on Logistics and SCM Research (RIRL 2010), BEM, September 29, 30 and October $1^{\text {st }}$.

Page, S.J. (2003), Tourism management: Managing for change. Oxford: ButterworthHeinemann.

Pellegrin-Romeggio, Frédéric. (2011), L’assemblage dynamique des chaînes logistiques multiservices : identification des compétences clés du pivot assembleur dans le contexte du voyage. Thèse de Doctorat en Sciences de Gestion, Université de la Méditerranée, AixMarseille II, soutenue le 13 Septembre.

Sinclair, M.T., Stabler, M. (1997), The economics of tourism. London: Routledge.

Tapper, R ; Font, X., (2004), Tourism supply chains, Report of a Desk Research Project for the Travel Foundation, from Leeds Metropolitan University for the travel foundation. Final Report, $31^{\text {st }}$ January.

Van der Yeught, C, (2009), «Favoriser l'émergence d'un acteur stratégique dans les destinations touristiques pour répondre aux défis du développement durable ", Cahier : L'organisation revisitée au travers du développement durable: une approche multidisciplinaire, No 26, Management \& Avenir, juin.

Yin, R.K. (2003), Case Study research. Design and Methods, Third Edition, VoL.5, Sage Publications.

Zhang, X., Song, H., Huang, G.Q. (2009), “Tourism Supply Chain Management: A New Research Agenda”, Tourism Management, Vol. 30, Issue 3, June, pp.345-358. 